\title{
Design of a High-Resolution High-Stability Positioning Mechanism for Crystal Optics
}

\author{
Deming Shu, Thomas S. Toellner, and Esen E. Alp \\ Advanced Photon Source \\ Argonne National Laboratory \\ Argonne, Illinois 60439, U. S. A.
}

\begin{abstract}
We present a novel miniature multi-axis driving structure that will allow positioning of two crystals with better than 50-nrad angular resolution and nanometer linear driving sensitivity. The precision and stability of this structure allow the user to align or adjust an assembly of crystals to achieve the same performance as does a single channel-cut crystal, so we call it an "artificial channel-cut crystal." In this paper, the particular designs and specifications, as well as the test results, for a two-axis driving structure for a high-energy-resolution artificial channel-cut crystal monochromator are presented.
\end{abstract}

\section{INTRODUCTION}

With the availability of third-generation of hard $\mathrm{x}$-ray synchrotron radiation sources, such as the Advanced Photon Source (APS) Argonne National Laboratory (ANL), $\mathrm{x}$-ray inelastic scattering and $\mathrm{x}$-ray nuclear resonant scattering provide powerful means for investigating vibrational dynamics of variety of materials and condensed matter systems.

The APS SRI-CAT 3-ID beamline is dedicated to high-energy-resolution $\mathrm{x}$-ray scattering studies in the energy range of $6-30 \mathrm{keV}$ [1]. A special 2.7-cm-period undulator, optimized for peak brilliance is installed. The beamline basic components include filters, white-beam slits, integral shutters, and the Kohzu double-crystal monochromator with water-cooled diamond crystals as a pre-monochromator. The beamline special components, such as custom-built high-energy-resolution monochromators and a dual-function (collimating or focusing) x-ray mirror system, provide a high flexibility for the optical system, so that users can optimize the beamline configuration for various applications. For instance, a 4-bounce highresolution monochromator using a nested channel-cut crystal approach was used to deliver a $\mathrm{x}$-ray beam with $\mathrm{meV}$ bandpass for $\mathrm{x}$-ray inelastic scattering experiments [2].

There are design restrictions to the nested channel-cut geometry. Because these two channel-cut crystals are nested within each other, the size of the channel-cut crystals becomes an important design factor. The availability of large crystals with good longrange crystallinity restricts the size of the outer channel-cut crystal. On the other hand, the input beam power absorbed by the first optical surface on the outer channel-cut 


\section{DISCLAIMER}

This report was prepared as an account of work sponsored by an agency of the United States Government. Neither the United States Government nor any agency thereof, nor any of their employees, make any warranty, express or implied, or assumes, any legal liability or responsibility for the accuracy, completeness, or usefulness of any information, apparatus, product, or process disclosed, or represents that its use would not infringe privately owned rights. Reference herein to any specific commercial product, process, or service by trade name, trademark, manufacturer, or otherwise does not necessarily constitute or. imply its endorsement, recommendation, or favoring by the United States Government or any agency thereof. The views and opinions of authors expressed herein do not necessarily state or reflect those of the United States Government or any agency thereof. 


\section{DISCLAIMER}

Portions of this document may be illegible in electronic image products. Images are produced from the best available original document. 
crystal can reach a fraction of a Joule and can cause crystal local temperature and strain variations. In certain high-energy-resolution applications, it becomes a major restriction to the optical design. To overcome these obstacles, we have developed a novel miniature multi-axis driving structure [3]. The precision and stability of this structure allow us to align or adjust an assembly of crystals to achieve the same performance as does a single channel-cut crystal, so we call it an "artificial channelcut crystal." Using this structure, we can make an outer channel-cut crystal large enough to optimize the nested monochromator's performance and compensate the crystal local temperature and strain variations for the two optical surfaces on the crystals.

\section{DESIGN SPECIFICATIONS}

The artificial channel-cut crystal was designed for a novel nested high-energyresolution monochromator, which is optimized for an ${ }^{151} \mathrm{Eu}$ nuclear resonance experiment at the energy of $21.542 \mathrm{keV}$. The high-energy-resolution monochromator uses a symmetric silicon (15 113) channel-cut crystal nested within an asymmetrically cut silicon (4 40 ) artificial channel-cut crystal. This optical design can produce a 1$\mathrm{meV}$ resolution beam with a high throughput. However, if we use a natural channelcut crystal to make the outer channel-cut crystal, the diffraction faces can be separated by $200 \mathrm{~mm}$, which makes it difficult to avoid problems associated with strain and temperature variations.

We use two asymmetrically cut silicon (4 40 ) crystals, which are each sized 25 $\mathrm{mm} \times 25 \mathrm{~mm} \times 50 \mathrm{~mm}$, to act as an artificial outer channel-cut crystal. The major specification for the artificial channel-cut crystal structure is the angular alignment capability between the two crystals. According to the 300-nrad Darwin width of the silicon $(440)$ in this asymmetrically cut geometry, a 50-nrad pitch alignment resolution is needed. Long-term positioning stability is also very important to the artificial channel-cut crystal. In a temperature-controlled environment $\left(+/-0.2^{\circ} \mathrm{C}\right)$, an angular drifting rate less than 25 -nrad per hour is required. Table 1 lists the design specifications for this $(440)$ artificial channel-cut crystal. Fig. 1 shows the artificial channel-cut crystal mounted on the Kohzu monochramator stage in the APS 3-ID-B experimental station.

\begin{tabular}{|ll|}
\hline TABLE 1. Design specifications for a $(4+0)$ artificial channel-cut crystal \\
\hline Maximum Overall Dimension & $216 \mathrm{~mm} \times 212 \mathrm{~mm} \times 92 \mathrm{~mm}$ \\
Main Shaft Diameter & $10 \mathrm{~mm}$ \\
Maximum Thickness in Nested Area & $30 \mathrm{~mm}$ \\
Single Crystal Size & $25 \mathrm{~mm} \times 25 \mathrm{~mm} \times 50 \mathrm{~mm}$ \\
Number of Angular Alignment Axis & 2 \\
Angular Alignment Resolution (Pitch) & $50 \mathrm{nrad}$ \\
Angular Alignment Resolution (Roll) & $600 \mathrm{nrad}$ \\
Angular Alignment Stability (Pitch) & Drift less than 25 nrad per hour \\
Angular Alignment Stability (Roll) & Drift less than 100 nrad per hour \\
Angular Alignment Range (Pitch) & 0.6 degree \\
Angular Alignment Rangc (Roll) & 2 degrce \\
\hline
\end{tabular}




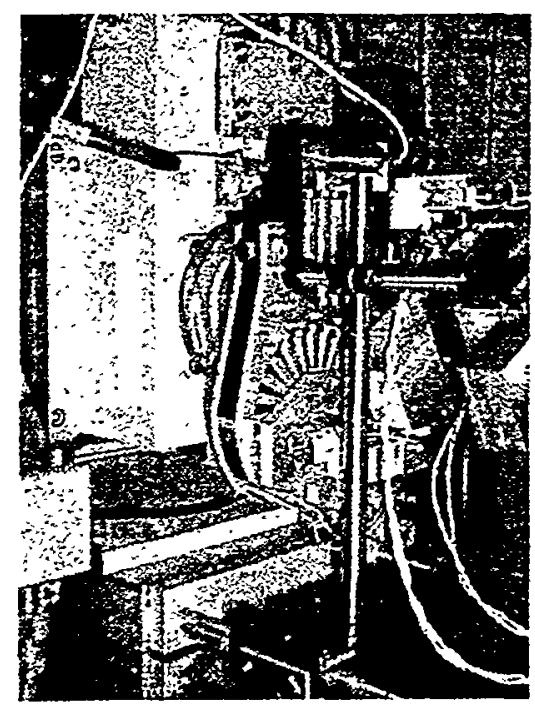

Figure 1. An artificial channel-cut crystal mounted on the Kohzu monochramator stage in the APS 3ID-B experiment station.

\section{HIGH-STIFFNESS WEAK-LINK MECHANISM DESIGN}

Designing such a miniature multi-axis driving structure with high stiffness and nanometer sensitivity is challenging. The novelty of this new structure is combining the closed-loop controlled piezoelectric transducer (PZT) technology with a novel high-stiffness weak-link design. Using stacked thin metal sheets configured and manufactured by chemical etching and lithography techniques, we were able to design a planar-shaped, high-stiffness, high-stability weak-link structure for artificial channel-cut crystal use (see Fig.2).

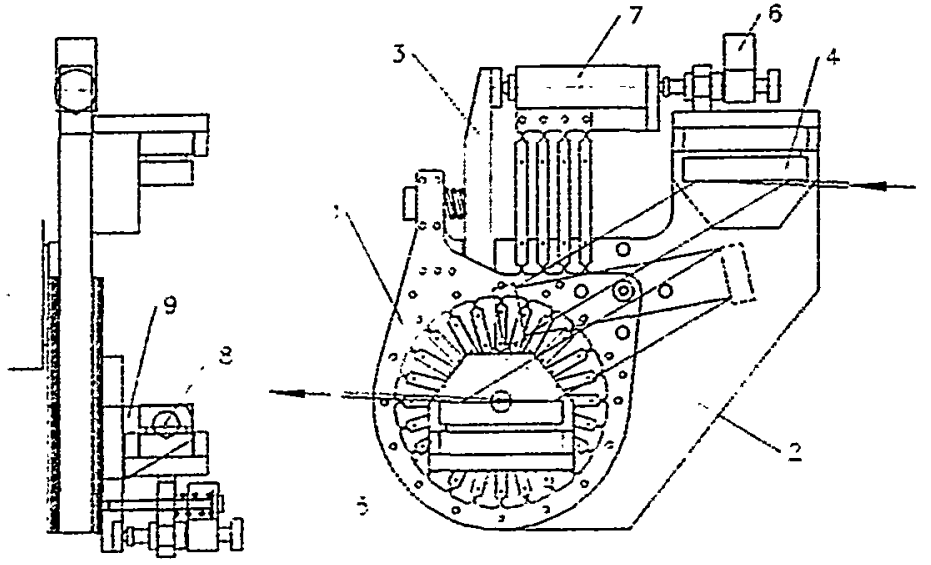

Figure 2. Design of the miniature multi-axis driving structure for an artificial channel-cut crystal. 
The structure consists of three sub-assemblies: one base structure and two crystal holders. The base structure includes a compact sine-bar driving mechanism for the crystal pitch alignment, which is the key component of the whole structure. There are two groups of stacked thin metal weak-link structures (1) mounted on each side of the base plate (2). A sine-bar (3) is installed on the center of the planar rotary shaft for the pitch alignment between the two $(4,40)$ single crystals $(4,5)$. Two linear drivers are mounted on the base plate serially to drive the sine-bar. The rough adjustment is performed by a Picomotor [5] (6) with a 20-nm to 30-nm step size. A Queensgate [6] closed-loop controlled PZT (7) with capacitance sensor provides 1-nm resolution for the pitch fine alignment. A pair of commercial flexure bearing (8) is mounted on one of the crystal holders (9), and a Picomotor driven structure (10) provides the roll alignment for the crystal.

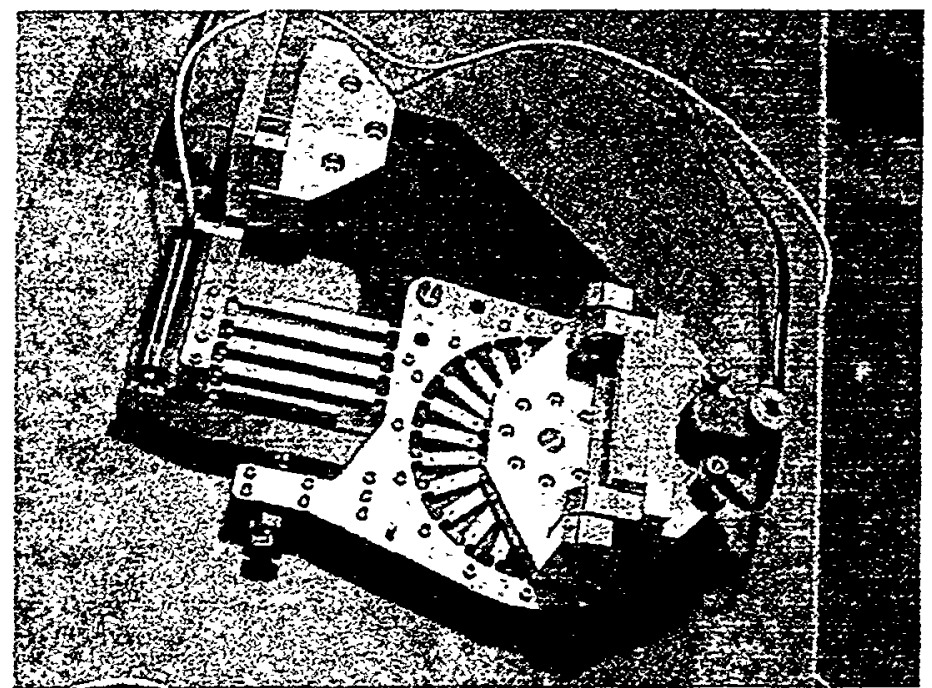

Figure 3. Photograph of the weak-link Structure mounted on the base plate.

Fig. 3 shows the shape of the metal weak-link sheet, which is produced by a photochemical machining process. Two motion structures are designed on the same metal sheet. A wheel-shaped weak-link group performs as a planar rotary shaft, and a parallelogram-shaped weak-link group acts as a linear slide. To optimize the system stiffness, we have chosen overconstrained mechanisms in this design. The precision of the modern photochemical machining process using lithography techniques make it possible to construct a strain-free (or strain limited) overconstrained mechanism on thin metal sheet. By stacking these thin metal weak-link sheets with align-pins, we can construct a solid complex weak-link structure with a reasonable cost. For our first prototype, $250-\mu \mathrm{m}$ thick stainless steel sheet was used. Each group consists of twenty weak-link sheets. 0.6 degree adjustment range was reached, which was agreed with the finite element analysis result. Fig. 4 shows a finite element simulation for the wheel-shaped weak-link displacement under a $0.89-\mathrm{Nm}$ torsion load. In this case, the maximum displacement on the weak-link is $94-\mu \mathrm{m}$, which is corresponding to a 0.25 degree angular motion on the planar shaft, and the maximum stress in the weak region is 175-Mpa. More analysis for this overconstrained mechanism will be discussed in a separate paper later. 


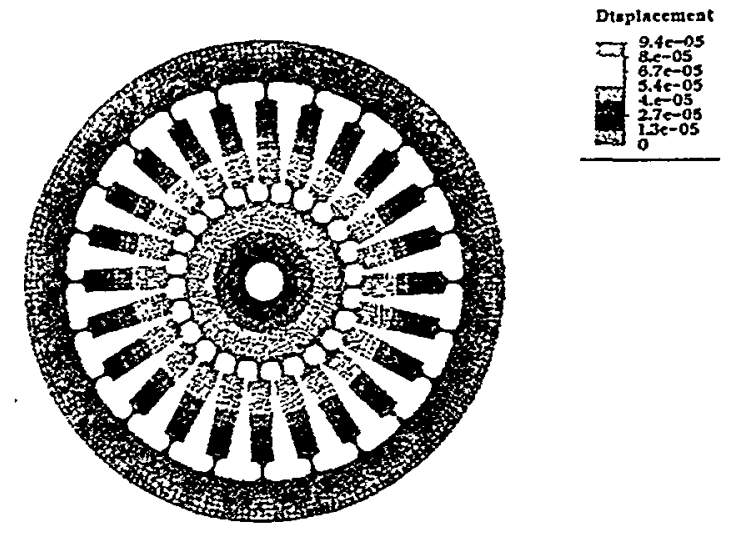

Figure 4. A finite element simulation for the wheel-shaped weak-link displacement under a $0.89-\mathrm{Nm}$ torsion load. The maximum displacement on the weak-link is $94-\mu \mathrm{m}$.

\section{SENSITIVITY TEST WITH LASER ENCODER}

We have tested the sensitivity of the weak-link sine-bar structure with a laser Doppler angular encoder. A 200-mm-long aluminum-arm is mounted on the center of the planar rotary shaft, perpendicular to the sine-bar. A set of prisms are mounted at the end of the arm as a multi-reflection displacement sensor [7]. During this test, a batch of 5-nm incremental steps are applied on the sine-bar by the Queensgate PZT. Fig. 5 shows a serial of angular steps recorded from the laser encoder with an averaging step size of 33 -nrad.

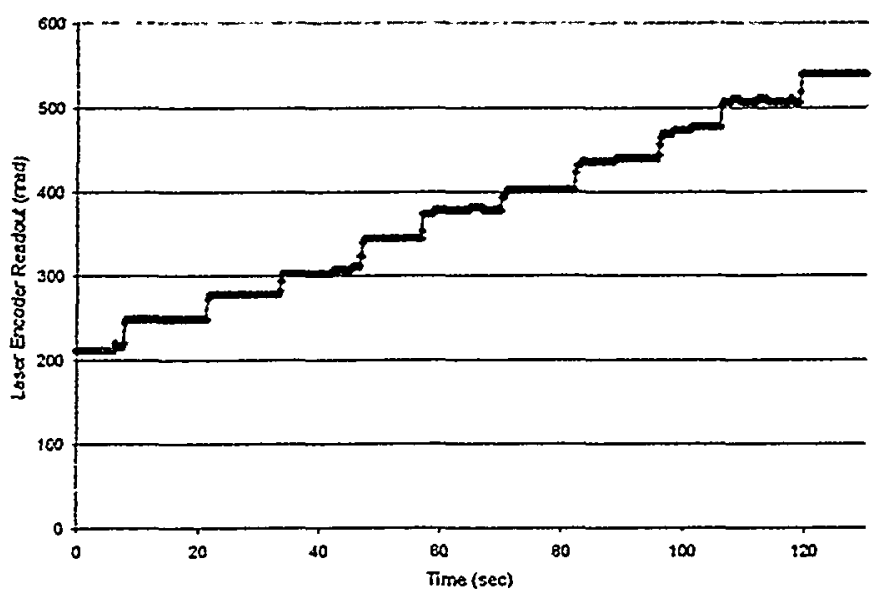

Figure 5. Sensitivity test of the weak-link sine-bar structure. The averaging step size is 33 -nrad.

\section{STABILITY RESULT FROM X-RAY EXPERIMENT}

We have tested the first prototype artificial channel-cut crystal as an outer crystal for a 4-bounce high resolution monochromator with nested configuration at the APS 
3-ID-B experimental station. As a typical case, Fig. 6 shows a two hours stability result with a $1-\mathrm{meV}$ bandwidth monochromatic beam.

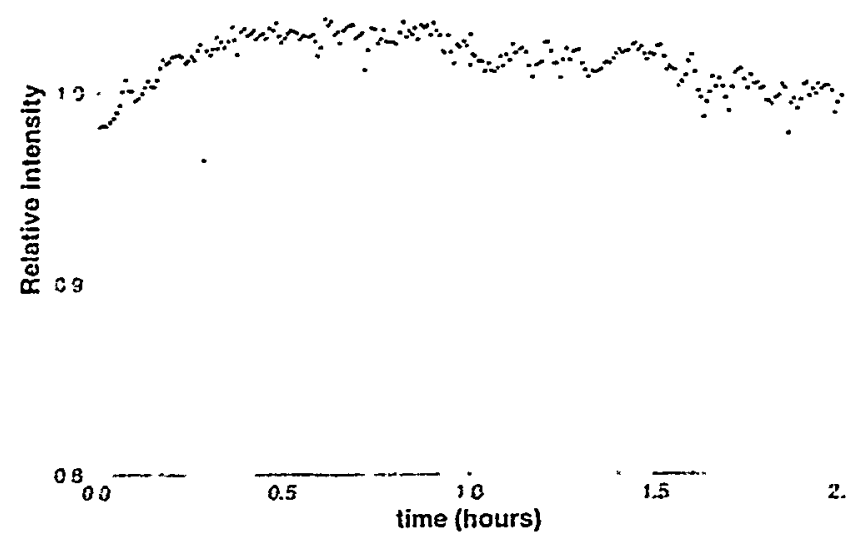

Figure 6. Srelative intensity measured by an ionization chamber after the high resolution monochromator as a function of time. The data is corrected for the decaying current in the storage ring.

\section{SUMMARY}

In the traditional channel-cut crystal design, the silicon is not only the material of choice for optics but also is the material for the base structure. The limitation of the silicon material mechanical properties and the difficulties of finishing the optical surfaces in certain geometries limited the design capability. An artificial channel-cut crystal will allow us to use the best materials for the base of the driving structures and use pre-finished crystals for optics to optimize the performance.

The benefit of an artificial channel-cut crystal lies in the reduction of the number of rotation axes of an optical setup, such as a monochromator. In certain cases, two or more crystals need to be rotated simultaneously, which is often difficult to achieve with high precision and good repeatability. By allowing independent motions of individual crystals, as described here, a great freedom in the design of the $\mathrm{x}$-ray optical setup is offered.

\section{ACKNOWLEDGMENTS}

We acknowledge help from Dr. Tuncer M. Kuzay, and Mr. Michel Lehmuller of the APS. This work was supported by the U.S. Department of Energy. Office of Sciences, under Contract No. W-31-109-Eng-38. 


\section{REFWRENCES}

1. E. E. Alp, Hyperfine Interactions, 1993

2 T S. Toellner, SPIE, 1992

3. D. Shu, T. S. Toellner. and E. E. Alp, Patent application in progress for ANL, Nov. 1998

t. T. S. Tocilner, to be published

5. Picomotor is a trade mark of Newfocus Co. California

6. Queensgate ia a trade mark of Queensgate Inc. Londion, England

7. D. Shu, E. E. Alp, J. Barraza, and T. M. Kuzay, Proceedings of SPIE, Vol.3429 (1998)284-292 